\title{
Joint Beamforming and Nullforming with PSO in Distributed MIMO :An Optimization Approach
}

\author{
Jagdish D. Kene ${ }^{1}$, Mridula S. Korde ${ }^{2}$, Deepali M. Kotambkar ${ }^{3}$ and Ajinkya P. Nilawar ${ }^{4}$ \\ ${ }^{1}$ Assistant Professor, Shri Ramdeobaba College of Engineering and \\ Management, Nagpur, Maharashtra, State, India \\ ${ }^{2}$ Associate Professor, Shri Ramdeobaba College of Engineering and \\ Management, Nagpur, Maharashtra State, India \\ ${ }^{3}$ Assistant Professor, Shri Ramdeobaba College of Engineering and \\ Management, Nagpur, Maharashtra State, India \\ ${ }^{4}$ Assistant Professor, Shri Ramdeobaba College of Engineering and \\ Management, Nagpur, Maharashtra State, India
}

\begin{abstract}
Distributed Multiple Input Multiple Output (DMIM0) systems pose significant challenges in implementation. The ones posed by distributed nullforming particularly call for special consideration. Our research interest is to treat the problem of multicasting a common message signal from a distributed array of wireless transceivers by beamforming to a set of beam targets, while simultaneously protecting a set of null targets by nullforming to them. In this work, it can be done by formulating the Joint beamforming and nullforming (JBNF) problem into an optimization problem. We introduce an adaptive algorithm in which each transmitter utilizes its own channel gains and the information gathered from a relay node that perfectly knows the channel responses between all the terminals. According to this, the transmitters and receivers, are designed for attempting to minimize the total transmit power subject to scalability constraints. Since this optimization problem is not convex, the use of the Particle swarm optimization (PSO) technique is proposed to find the optimum solution. The proposed method does not increase the network overheads with the number of transmitters which yields the proposed system design more efficient.
\end{abstract}

KEY WORDS: BEAMFORMING, NULLFORMING, MIMO, PSO, SINR.

\section{INTRODUCTION}

With the speedy development of communication technologies, wireless networks have emerged as a vital part of the modern society (Hameed and Khawar). This increases the demand for high voice, data speeds and reduces the network charges, however the increase in

\section{ARTICLE INFORMATION}

*Corresponding Author: kenejd@rknec.edu

Received 18th Oct 2020 Accepted after revision 24th Dec 2020

Print ISSN: 0974-6455 Online ISSN: 2321-4007 CODEN: BBRCBA

Thomson Reuters ISI Web of Science Clarivate Analytics USA and Crossref Indexed Journal

\section{Clarivate
Analytics}

NAAS Journal Score 2020 (4.31)

A Society of Science and Nature Publication,

Bhopal India 2020. All rights reserved.

Online Contents Available at: http//www.bbrc.in/

Doi: http://dx.doi.org/10.21786/bbrc/13.14/78 system interference caused as a result of the inadequate broadcast spectrum which degrades the signal quality and brings the essentiality to familiarize aubiquitous technology namely Beamforming (H. Dai et. al.). Beamforming can be utilized both within the transmitting and the receiving end to attain the spatial selectivity (D. Palomar et.al.). It adjusts the antenna weights to mitigate fading channel or interference effects, to enhance the characteristic of the signal (H. Bejar et. al.). The beamforming is attained by maximizing the output power of the array within the path of the desired signal and minimizing the output authorization of the antenna array within the track of the interfering signal. The interfering signal is minimized by the course of inserting nulls within the explicit track called null forming (R. Farrokh et. al.). 
Joint beam forming and null forming (JBNF) are spatial filtering techniques wherein a group of dispersed transmitters in a wireless network graciously transmits the nonentity message signal to the other nodes within the network (A. Kumar). JBNF furthermore has the characteristic of better scalability, excessive energy efficiency, asymptotic scalability, noise without drift, etc. (M. Rahman et. al.) (R. Bitmead). A MIMO (Multiple Input Multiple Output) channel is a component of spectral efficient technology can be constructed as a matrix with Independent and Identically Distributed (IID) hang-up Gaussian entries, which will furnish multiple spatial dimensions for single communications (S. Zhang et.at.). Distributed MIMO (D-MIMO) will also be in a position to represent the generalization of Distributed Antenna Systems (DAS) (H. Zhang et. al.) The dispersed MIMO is not only handy for wireless sensor networks in the wireless channel but also used for networks of solitary antenna nodes (S. Jafar et al.). D-MIMO system integrates the precedence of point-to-point MIMO and DAS, and has the capability to utilize mutually the spatial micro and macro diversities (D. Wang). Recently D-MIMO can be deed as a remedy for the indiscriminate paradigm for multiple antenna communications (H. Dai et.al.).

There are many algorithms adapted to find the join beam forming and null forming problem analogous to the method proposed in (H. Chen et.al.) which can introduce the incorporation of antenna array beamforming with multi user detection which is used to toughen the detection efficiency for wireless communications under multipath interference. The method proposed in (W. Wei et. al.) provides an algorithm for null forming. This algorithm can effectively improve the performance of antenna pattern through a crafty transformation of array directing vectors and signal correlation matrix respectively. In an adaptive beamforming algorithm is proposed with the aid of utilizing the Minimum Mean Square Error (MMSE), power minimization and maximum Signal to Interference plus Noise Ratio (SINR) (N. Noordin et. al.).

The scheme projected in (Y. Eldar et.al.) is employed to discuss the snag of beamforming for signal estimation which is used to analyze the signal amplitude and resulted as the estimated consequential signal which is far from the true signal. The null forming algorithm with auditory sub bands have introduced in (H. Zhang et. al.) is intended with an infinite duration impulse response (IIR) filter to correlate the disparity of the microphone pair. The scalable algorithm designed in (A. Kumar et. al.) typically aim at minimizing the mean square error (MSE) but not on maximizing the signal to Interference plus noise ratio (SINR). It is important to design an efficient algorithm which overcomes the problem of multicasting with high SINR and reduced MSE and it is challenging in distributed MIMO systems. On these considerations we set the following as goals in this research work:

- To consider the JBNF problem in distributed MIMO with the objective of maximizing SINR and minimizing the MSE between the achieved and desired modulating amplitudes at the receivers.

- To introduce an adaptive algorithm in which each transmitter utilizes its own channel gains and the information gathered from a relay node that knows perfectly all the channel responses between all the terminals.

- $\quad$ To use Particle swarm optimization (PSO) techniques to minimize the total transmit power subject to scalability constraints.

- The remainder of this research paper is outlined as follows: Section 2 presents an overview of deep knowledge of the proposed work. The simulation results of the proposed work is presented in section 3 followed by the conclusion of the proposed work in section 4.

2. Optimal Joint Beamforming and Nullforming in Distributed MIMO: In this section we have presented the JBNF problem statement with the system model and the optimal solution suggested for the same.

a. System Model \& Assumptions: Let we consider the problem of distributed joint beamforming and nullforming as shown in figure 1 . The system with $\mathrm{P}$ number of transmitters and $Q$ number of receivers with $1,2, \ldots . Q_{1}$ beam targets and $Q_{1}+1 \ldots \ldots . . Q$ null targets are considered in this research work. Let we consider xij is the complex gain from the ith transmitter to the jth receiver and $\mathrm{xj}$ be the $\mathrm{jth}$ column of $\mathrm{X}$, corresponding to the channel vector to receiver $\mathrm{j}$ then the $\mathrm{P} \times \mathrm{Q}$ channel matrix can be defined as $\mathrm{X}=\left[\begin{array}{lll}\mathrm{x}_{1} & \mathrm{x}_{2} & \ldots . . . . \mathrm{xQ}\end{array}\right]$. Each single antenna in the transmitter side has to broadcast a common message signal $\mathrm{M}_{\mathrm{s}}$ targeting each single antenna in the receiver side and forming nulls at another set of receivers in parallel.

Figure 1: JBNF Problem

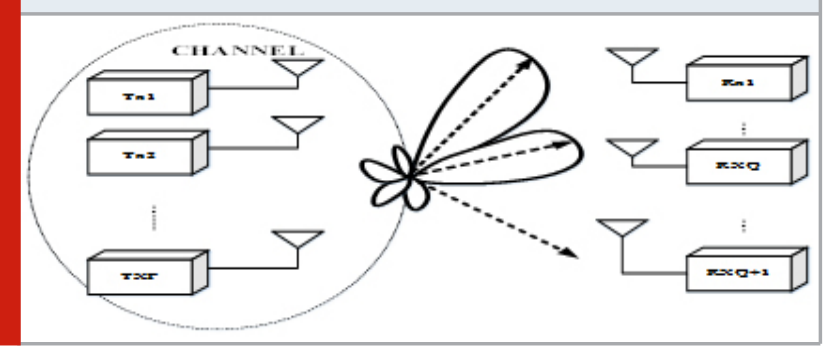

The transmitters form a virtual antenna array and choose phases and amplitudes to shape the array's pattern such that beams and nulls are created at desired locations. By simultaneously transmitting beams and nulls, coherent combining gains can be achieved toward intended receivers while protecting unintended receivers.

b. Problem Statement: Designing a novel algorithm that overcomes the problem of multicasting with high SINR and reduced MSE is challenging in distributed MIMO systems. Conventional scalable algorithms typically aim at minimizing the mean square error (MSE) but not on maximizing the signal to interference noise ratio (SINR). It can be done by formulating the JBNF problem into an 
optimization problem. So that the primary objective of this work is set to:

$$
\left\{A(t)=\max [\operatorname{SINR}(w)]+\max \left[\frac{1}{M S E\{A(t)}\right]\right.
$$

Where amplitude of the input signal is $A(t)$. In addition to that in this work we aim to adapt $\delta[k]$ in a distributed fashion so that $\delta^{x}[k] x_{j}$ are driven towards nonzero values $\sigma_{j}$

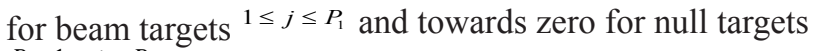
$P_{1}+1 \leq j \leq P \underset{\text { Set }}{ } \sigma_{j}=0, \forall j\left\{P_{1}+1, \ldots, P\right\}$ and $\sigma=\left[\sigma_{1}, \ldots, \sigma_{P}\right]^{T}$ So that the quadratic cost function can be defined as:

$$
C_{w}=\|\psi[k]-\sigma\|^{2}=\left\|X^{X} \delta[k]-\sigma\right\|^{2}
$$

In order to reduce the quadratic cost function, the JBNF problem can be rewritten by accommodating noise as follows:

$$
\begin{aligned}
C_{w}(\delta) & =E_{w}[\| \psi-\sigma \\
& =E_{w}\left[\| X^{X} \delta .\right.
\end{aligned}
$$

Minimum value of Eqn. (3) is equivalent to

$$
\min C_{w}=\left|X^{X} \delta-\sigma\right|^{2}
$$

The JBNF problem was made more convex by Particle Swarm Optimization (PSO) algorithm, which is inspired by bird group behavior (Zhang et. al.). The PSO algorithm is easy to implement and more efficient. It has been widely used in multi objective optimization, pattern recognition, signal processing and other fields. It has faster computing speed and good global search capability. Owing to this, it is particularly suitable for large scale mathematical optimization problems. This algorithm also has adaptive capability and it is useful for solving optimization problems in dynamic environments. In the PSO algorithm, each particle is viewed as a no weight and volumetric dot in d dimensional search space, and flies at a certain speed. Assumption that one group has $\mathrm{N}$ particles, $\mathrm{Xi}$ is particle of i's current position, $\mathrm{Vi}$ is particle of i's current flight speed, Pbesti is particle of i's best experienced position, Gbestn is group's best location. Each particle's flight speed and direction are determined by its position and velocity. From these parameters, an optimized fitness function determines solution's merits. The standard PSO algorithm can be described as:
$X_{i}(k+1)=X_{i}(k)+V_{i}(k+1), i=1,2, \ldots . N$

(6)
Figure 2: PSO Optimization

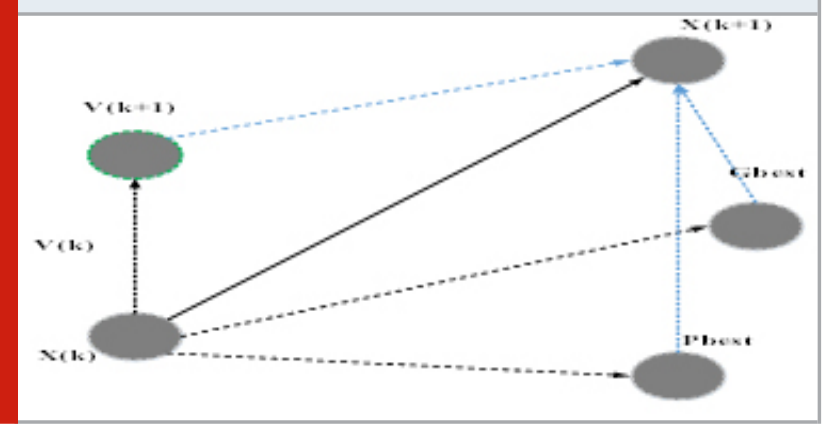

Where rand $_{1}($.$) and rand.( are the random numbers$ ranging from 0 to $1, c_{1}$ and are the weighting factors, is the inertia weight $\bar{\omega}$. At the point, all particles that fly over the D-dimensional solution space are liable to modernized rules for new positions, awaiting the global optimal position is established. Figure 2 demonstrates an exploration method of PSO using the velocity and location appraise rules given in (4) and (5). The inertia weight can be calculated as:

$$
\varpi=\varpi_{\max }-\frac{\varpi_{\max }-\varpi_{\min }}{I_{\max }} \times I
$$

Where $\overline{\boldsymbol{m}}_{\max }, \overline{\boldsymbol{\sigma}}_{\min }$ is the lower and upper inertia weights $I_{\max }$, is the highest number of iteration, is the present iteration $I$. With all the above constraints the overall objective function for PSO is defined as:

With all the above constraints the overall objective function for PSO is defined as:

$$
O F(P S O)=\min \left[C_{w}\right]+\max \left[\frac{\operatorname{SINR}(w)}{\operatorname{MSE}\{A(t)\}}\right]
$$

In this paper, authors concentrate on achieving both beam targets and null targets which differentiates our work from most of the conventional approaches.

c. Adaptive Algorithm for JBNF: To achieve the desired goal to adopt $\delta[k]$ in a distributed fashion while in JBNF we introduce an adaptive algorithm in which each transmitter utilizes its own channel gains $G_{c}$ and the information gathered from a relay node that perfectly knows the channel responses between all the terminals. The algorithmic steps of the proposed adaptive algorithm to solve the JBNF problem are explained in algorithm1.

The proposed adaptive algorithm adapts $\delta[k]$ at slot $\mathrm{k}+1$, only by the knowledge gathered from the relay 
node and by its own channel gain. In specific to adopt $\delta[k]$, transmitter i does not need the channel gains of other transmitters which improves the performance of the proposed algorithm, thus reduces the computational complexity.

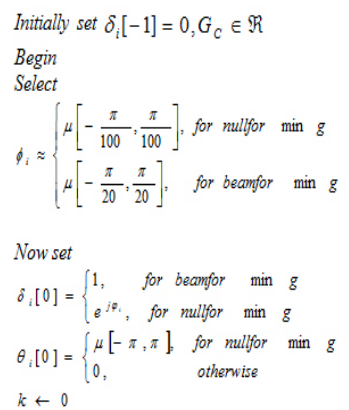

Algorithml: Adaptive Algorithm for JBNF

3. Simulation Results: In this section, we have presented the simulation results of the proposed work which is implemented and validated using MATLAB. We consider a JBNF system with 40 transmitters and 5 receivers of which 2 are beam targets and the remaining 3 receivers are null targets. All channel gains are modeled in the range of $(0,1)$, and the noise level is taken to be -40 $\mathrm{dB}$ at each receiver. We encode the real and imaginary parts of source signal into double precision floating point numbers with each requiring 64 bits for a total feedback of 128 bits per iteration.

The beam formed during the transmission converges at an optimum level which is shown in figure 3. From figure 3, it is observed that the optimum beam formed in the range of -900 to +900 . With the objective of minimizing MSE, the optimum beam formed during data transmission in MIMO converges at a minimum level. Figure 4 shows the performance comparison of the proposed work in terms of received signal power obtained for all the five receivers present in reception side. The similar simulation environment and setup is used to perform this comparison. Figure 4(a) shows the variation of received signal power with $\mathrm{Nt}=2, \mathrm{Nt}=3$ and $\mathrm{Nt}=4$ respectively. We observe that for 6 bits, the curves overlap for receiver 3 , receiver 4 and receiver 5 furthermore leads to an error floor. Figure 4(b) shows the variation of received signal power with delay $=1 \mathrm{e}-3$, $2 \mathrm{e}-3$ and $3 \mathrm{e}-3$ respectively. Comparing the results of the five receivers, we see that the introduction of an interference with receivers 3, 4 and 5 operating with 6 bits results in a similar MSE values as that of a receiver without optimization. Considering that the above setup reduces the interfere contributions, these results suggest that the proposed system with optimization can perform close to theoretical MIMO while consuming a fraction of the power compared to other existing works presented without optimization.
Figure 3: Optimum Beam Forming

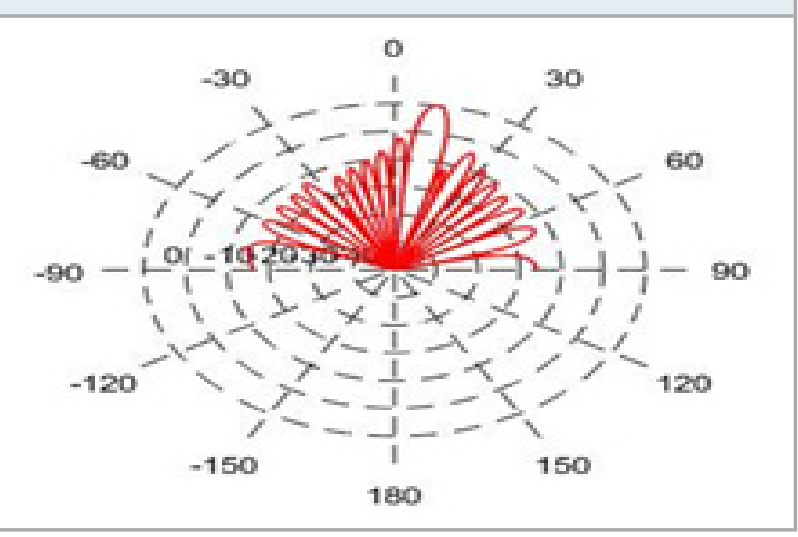

Figure 4: Performance Comparison (a) Received signal power with $\mathrm{Nt}=2,3$ and 4 respectively (b) Received signal power with varying delay

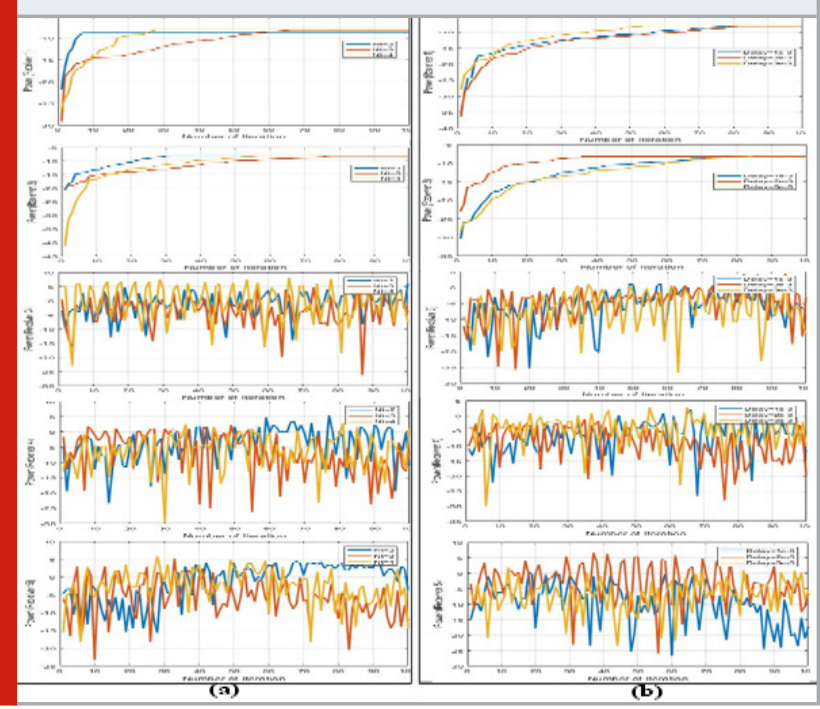

\section{CONCLUSION}

A novel distributed joint beamforming and nullforming based on PSO algorithm was proposed in this paper. Our algorithm achieves simultaneous beams and nulls in a quadratic framework guaranteeing rapid convergence. The proposed work aims at maximizing the SINR with minimizing MSE. It scales to large transmit arrays, as it only requires aggregate broadcast feedback from the receivers and the convergence rate improves with the number of transmitters. The optimum beamformed by PSO ensures the fulfilment of the objective of this work. The simulation results enclosed in this paper also proves the significance of the proposed work.

\section{REFERENCES}

A. Kumar, "Scalable algorithms for distributed beam forming and null forming," 2017 The University of Iowa.

A. Kumar, R. Mudumbai, S. Dasgupta, U. Madhow, D. 
Brown, "Distributed MIMO Multicast with Protected Receivers: A Scalable Algorithm for Joint Beamforming and Nullforming." 2017 IEEE Transactions on Wireless Communications, vol. 16, no.1, 2017, pp. 512-252.

C. Yee, and S. Kumar, "Sensor networks: evolution, opportunities, and challenges," 2003 Proceedings of the IEEE, pp. 1247 - 1256. DOI: 10.1109/ JPROC.2003.814918.

D. Wang, "Spectral efficiency of distributed MIMO systems," 2013 IEEE Journal on Selected Areas in Communications, vol. 31, no. 10, 2013, pp. 2112 - 2127. DOI:10.1109/JSAC.2013.131012.

D. Palomar, J. Cioffi, M. Lagunas, "Joint Tx-Rx beamforming design for multicarrier MIMO channels: A unified framework for convex optimization,” 2003 IEEE Transactions on Signal Processing, vol. 51, no. 9, 2003, pp. 2381 - 2401. DOI: 10.1109/TSP.2003.815393.

Hameed, Khawar, "The application of mobile computing and technology to health care services," 2003 Telematics and Informatics, vol. 20, no. 2.

H. Dai, K. Ning, W. Ng, M. Li, M. Wu, "An overview of using directional antennas in wireless networks," 2013 International Journal of Communication Systems, vol. 26, No. 4.

H. Bejar, S. Zazo, D. Palomar, "Energy efficient collaborative beamforming in wireless sensor networks," 2014 IEEE Transactions on Signal Processing, vol. 62, no. 2, 2014, pp. 496-510.

H. Zhang and D. Huaiyu, "On the capacity of distributed MIMO systems," 2004 In Proceeding of Conference of Information Sciences and Systems (CISS).

H. Dai, Z. Hongyuan, Z. Quan, "Some Analysis in Distributed MIMO Systems,” 2007 JCM Z, vol. 2, no. 3, 2007.

H. Chen and J. Lee, "Adaptive joint beamforming and B-MMSE detection under multipath interference," 2004 IEEE Proceedings on Communications, 2004, pp. 605612. DOI: $10.1049 /$ ip-com:20040578.
H. Zhang, F. Qiang, Y. Yonghong, "Adaptive nullforming algorithm with auditory sub-bands," 2006 Lecture notes in computer science, vol. 4274, 2006, pp.248-272.

M. Rahman, S. Dasgupta and R. Mudumbai, "A Scalable Feedback-Based Approach to Distributed Null forming," 2013 In WiCON, pp. 78-84.

N. Noordin and K. Zulkeflee, "Beamforming algorithms for adaptive array antenna,” 2014 In 2nd International Conference on Electronic Design (ICED), 2014.

R. Farrokh, L. Tassiulas, K Liu, “Joint optimal power control and beamforming in wireless networks using antenna arrays," 1998 IEEE transactions on communications, vol. 46, no. 10. 1998, pp. 751-761. https://doi.org/10.1155/S1110865704311030.

R. Bitmead, "Convergence in distribution of LMS-type adaptive parameter estimates," 1983 IEEE Transactions on Automatic Control, vol. 28, no. 1.

S. Zhang, J. Ni, Q. Peng, "Joint beamforming and power control algorithm for cognitive MIMO broadcast channels via game theory" 2017 EURASIP Journal on Wireless Communications and Networking, no. 1. DOI 10.1186/s13638-017-0918-z.

S. Jafar, and S. Shlomo, "Degrees of freedom region of the MIMO \$ X \$ channel,” 2008 IEEE Transactions on Information Theory, vol. 54, no. 1. https://doi. org/10.1109/TIT.2007.911262.

W. Wei, H. Ma and M. Sun, "An Improved Adaptive Null Forming Algorithm in Wireless Communication,” 2006 First International Conference on Communications and Networking in China, ChinaCom'06, 2006.

Y. Eldar, N. Arye, S. Patricio, "A competitive meansquared error approach to beamforming," 2007 IEEE Transactions on Signal Processing, vol. 55, no. 11, 2007, pp. 5143-5154. DOI: 10.1109/TSP.2007.897883.

Zhang, C. M. Wu, L. Linlin, “An optimal PSO distributed precoding algorithm in QRD-based multi-relay system," 2013 Future Generation Computer Systems, vol. 29, no. 1, 2013, pp. 161-171. 